\title{
RECENT DEVELOPMENTS IN THE THEORY OF ANDERSON MODULES
}

\author{
BRUNO ANGLÈS, TUAN NGO DAC, AND FLORIC TAVARES RIBEIRO
}

\begin{abstract}
Let $K$ be a global function field over a finite field of characteristic $p$ and let $A$ be the ring of elements of $K$ which are regular outside a fixed place of $K$. This report presents recent developments in the arithmetic of special $L$-values of Anderson $A$-modules. Provided that $p$ does not divide the class number of $K$, we prove an "analytic class number formula" for Anderson $A$-modules with the help of a recent work of Debry. For tensor powers of the Carlitz module, we explain how to derive several log-algebraicity results from the class number formula for these Anderson modules.
\end{abstract}

\section{Contents}

\begin{tabular}{lr}
\hline Introduction & 1 \\
\hline $1 . \quad$ Background & 2 \\
\hline 2. Anderson modules & 4 \\
\hline 3. Arithmetic of Anderson modules & 7 \\
\hline 4. The class formula à la Taelman & 9 \\
\hline $5 . \quad$ An example: tensor powers of the Carlitz module & 13 \\
\hline $6 . \quad$ Further reading on related topics & 15 \\
\hline References & 17
\end{tabular}

\section{INTRODUCTION}

A classic topic in number theory is the study of the Riemann zeta function $\zeta($.) and its special values $\zeta(n)$ for $n \in \mathbb{Z}$. In 1935, Carlitz suggested to transport the classical results to the function field setting in positive chracteristic. In [19], he considered the rational function field equipped with the infinity place and introduced the Carlitz zeta values $\zeta_{A}(n)$ which are considered as the analogues of $\zeta(n)$. They are related to the so-called Carlitz module which is the first example of a Drinfeld module.

2010 Mathematics Subject Classification. Primary 11G09; Secondary 11M38, 11R58.

Key words and phrases. Drinfeld modules, Anderson modules, $L$-series in characteristic $p$, class formula, log-algebraicity. 
In 1970s, Drinfeld 25, 26] invented many fundamental objects including Drinfeld modules. These objects are associated to any function field equipped with an arbitrary place. In 1986, the story continued with Anderson [1] who developed the theory of Drinfeld modules in higher dimensions. Over the last fifty years, the arithmetic study of Drinfeld modules and Anderson modules has played a dominant role in function field arithmetic.

In recent years, the theory of special $L$-values of Anderson modules has rapidly developed due to the fundamental works of Taelman [54] and Pellarin [48. The present paper aims to report recent developments in this topic. In particular, we focus on the theory of the number class formula à la Taelman and its possible connection with that of log-algebraicity.

\section{Outline.}

The paper is organized as follows.

- In Section 1, we fix some notation and give basic definitions of the Goss map and the co-volumes of lattices.

- In Section 2, we introduce the notion of Anderson modules and provide a number of examples.

- In Section 3, we study Anderson modules defined over a finite extension of our function field. We introduce several fundamental objects such as the unit module and the class module defined by Taelman as well as the module of Stark units.

- In Section 4, we study the arithmetic of Anderson modules. In Section 4.1. we state a conjecture on the class formula à la Taelman for Anderson modules and report what is known about this conjecture. In Section 4.2. we explain a key result due to Debry in 2016 and establish the main result of this paper which proves Conjecture 4.1 under a mild condition (Theorem 4.4.

- In Section 5, we provide an application of the class formula given in our recent paper [1]. We investigate tensor powers of the Carlitz module and derive some log-algebraicity results from the class formula for these Anderson modules, which generalize the fundamental work of Anderson and Thakur [7.

- In Section 6, we briefly give an (incomplete) list of references for related topics.

\section{Acknowledgements.}

Part of this work was done during the authors' visit to Vietnam Institute for Advanced Study in Mathematics (VIASM) in June-August 2018. We are grateful to VIASM for its hospitality and great working conditions.

The second author (T. ND.) was partially supported by ANR Grant PerCoLaTor ANR-14-CE25-0002.

\section{BACKGROUND}

This section follows closely the presentation given in [10, Section 2.3, 31, Section 8.2 and [54], Proposition 4. 


\subsection{Notation.}

Throughout this paper, $K$ denotes a global function field of genus $g$ over a finite field $\mathbb{F}_{q}$ of characteristic $p$, having $q$ elements $\left(\mathbb{F}_{q}\right.$ is algebraically closed in $\left.K\right)$. We fix a place $\infty$ of degree $d_{\infty} \geq 1$ of $K$ and denote by $A$ the ring of elements of $K$ which are regular outside $\infty$. The $\infty$-adic completion $K_{\infty}$ of $K$ is equipped with the normalized $\infty$-adic valuation $v_{\infty}: K_{\infty} \rightarrow \mathbb{Z} \cup\{+\infty\}$ and has residue field $\mathbb{F}_{\infty}$. The completion $\mathbb{C}_{\infty}$ of a fixed algebraic closure $\bar{K}_{\infty}$ of $K_{\infty}$ comes with a unique valuation extending $v_{\infty}$, it will still be denoted by $v_{\infty}$. We define the Frobenius $\tau: \mathbb{C}_{\infty} \rightarrow \mathbb{C}_{\infty}$ as the $\mathbb{F}_{q}$-algebra homomorphism which sends $x$ to $x^{q}$.

We fix $\pi \in K_{\infty}$ such that $v_{\infty}(\pi)=1$ and $\pi^{\prime} \in \bar{K}_{\infty}$ such that $\pi^{\prime d_{\infty}}=\pi$. Finally, we choose a sign function sgn : $K_{\infty}^{\times} \rightarrow \mathbb{F}_{\infty}^{\times}$, that is, a group homomorphism such that $\left.\operatorname{sgn}\right|_{\mathbb{F}_{\infty}^{\times}}=\operatorname{Id}_{\mathbb{F}_{\infty}^{\times}}$and $\operatorname{sgn}(\pi)=1$.

Example 1.1 (The basic genus 0 case).

Our basic example deals with the case when $g=0$ and $d_{\infty}=1$. It is called the basic genus 0 case or the case $A=\mathbb{F}_{q}[\theta]$.

Let $K=\mathbb{F}_{q}(\theta)$ be the rational function field with an indeterminate $\theta$. Let $\infty$ be the infinity place of degree $d_{\infty}=1$. Then $A=\mathbb{F}_{q}[\theta]$ and $K_{\infty}=\mathbb{F}_{q}\left(\left(\frac{1}{\theta}\right)\right)$ equipped with the discrete valuation $v_{\infty}$. We choose $\pi=\frac{1}{\theta}$ and observe that $v_{\infty}(\theta)=-1$. For $d \in \mathbb{N}, A_{+, d}$ denotes the set of monic elements in $A$ of degree $d$. Note that $A$ is a principal ideal domain.

Example 1.2 (Principal ideal domains).

Another important class of rings $A$ consists of principal ideal domains. In particular, $d_{\infty}=1$. Apart from the polynomial ring $A=\mathbb{F}_{q}[\theta]$ mentioned above, there are only four other rings. We extract the list from the article of Thakur [56].

- Example A: $A=\mathbb{F}_{3}[x, y] /\left(y^{2}-x^{3}+x+1\right)$ for which $g=1$.

- Example B: $A=\mathbb{F}_{4}[x, y] /\left(y^{2}+y-x^{3}-\alpha\right)$ for which $g=1$. Here $\alpha$ is an element of $\mathbb{F}_{4}$ satisfying $\alpha^{2}+\alpha+1=0$.

- Example C: $A=\mathbb{F}_{2}[x, y] /\left(y^{2}+y-x^{3}-x-1\right)$ for which $g=1$.

- Example D: $A=\mathbb{F}_{2}[x, y] /\left(y^{2}+y-x^{5}-x^{3}-1\right)$ for which $g=2$.

\subsection{The Goss map.}

We observe that $A$ is a Dedekind domain. Let $\mathcal{I}(A)$ be the group of non-zero fractional ideals of $A$ and let $\mathcal{P}(A)=\left\{x A, x \in K^{\times}\right\} \subset \mathcal{I}(A)$. We set $\operatorname{Pic}(A)=\frac{\mathcal{I}(A)}{\mathcal{P}(A)}$. Recall that if $\mathrm{Cl}(K)$ denotes the group of classes of degree zero divisors of $K$, we have an exact sequence of finite abelian groups

$$
0 \rightarrow \mathrm{Cl}(K) \rightarrow \operatorname{Pic}(A) \rightarrow \frac{\mathbb{Z}}{d_{\infty} \mathbb{Z}} \rightarrow 0 .
$$

Let $\operatorname{deg}: \mathcal{I}(A) \rightarrow \mathbb{Z}$ be the group homomorphism given by

$$
\operatorname{deg}(I):=\operatorname{dim}_{\mathbb{F}_{q}}(A / I), \quad \text { for } I \in \mathcal{I}(A), I \subset A .
$$

We set $\operatorname{deg}(x):=\operatorname{deg}(x A)$ and observe that

$$
\operatorname{deg}(x A)=-d_{\infty} v_{\infty}(x), \quad \text { for } x \in K^{\times} .
$$


Let $I \in \mathcal{I}(A)$, then there exists an integer $h \geq 1$ such that $I^{h}=x A$ for some $x \in K^{\times}$. Let $u$ be the unique element in $\bar{K}_{\infty}$ such that $v_{\infty}(u-1)>0$ and

$$
u^{h}=\frac{x \pi^{-v_{\infty}(x)}}{\operatorname{sgn}(x)} .
$$

Following Goss ([31, Section 8.2), we set

$$
[I]:=u \pi^{\prime-\operatorname{deg}(I)} .
$$

One can show that the map [.] $: \mathcal{I}(A) \rightarrow \bar{K}_{\infty}^{\times}$is a group homomorphism (see [31, Section 8.2). Further, we have

$$
[x A]=\frac{x}{\operatorname{sgn}(x)}, \quad \text { for } x \in K^{\times} .
$$

Let $M$ be a finite $A$-module, then there exist non-zero integral ideals $I_{1}, \ldots, I_{n}$ of $A$ such that there is an isomorphism of $A$-modules

$$
M \simeq \prod_{j=1}^{n} \frac{A}{I_{j}}
$$

We define

$$
[M]_{A}:=\left[I_{1} \cdots I_{n}\right]
$$

If we have an exact sequence of finite $A$-modules $0 \rightarrow M_{1} \rightarrow M_{2} \rightarrow M_{3} \rightarrow 0$, then

$$
\left[M_{2}\right]_{A}=\left[M_{1}\right]_{A}\left[M_{3}\right]_{A} .
$$

\subsection{Lattices and co-volumes.}

Let $L / K$ be a finite extension of fields and let $V$ be a finite dimensional $K_{\infty^{-}}$ vector space. Let $M$ be an $A$-submodule of $V$. We say that $M$ is an $A$-lattice in $V$ if $M$ is discrete and cocompact in $V$.

If $M, N$ are two $A$-lattices in $V$, then one can show that there exists an isomorphism of $K_{\infty}$-vector spaces $\sigma: V \rightarrow V$ such that $\sigma(M) \subset N$ ([10, Lemma 2.8). The co-volume of these lattices is defined by

$$
[M: N]_{A}:=\frac{\operatorname{det}_{K_{\infty}} \sigma}{\operatorname{sgn}\left(\operatorname{det}_{K_{\infty}} \sigma\right)}\left[\frac{N}{\sigma(M)}\right]_{A}^{-1} .
$$

One can show that $[M: N]_{A}$ does not depend on the choice of $\sigma$ ([10, Lemma 2.9). Furthermore, if $N \subset M$, then we have

$$
[M: N]_{A}=\left[\frac{M}{N}\right]_{A} .
$$

\section{Anderson modules}

\subsection{Definitions.}

Let $d \geq 1$ be an integer. For an $\mathbb{F}_{q^{-}}$-algebra $B$, let $M=\left(a_{i j}\right)_{1 \leq i, j \leq d} \in M_{d \times d}(B)$ be a $d \times d$ matrix with coefficients in $B$. If $k \geq 0$ is an integer, we set $M^{(k)}$ to be the matrix whose $i j$-entry is given by $\left(a_{i, j}\right)^{(k)}:=\left(a_{i, j}^{q^{k}}\right)$. We denote by $M_{d \times d}(B)\{\tau\}$ the non-commutative ring of twisted polynomials in $\tau$ with coefficients in $M_{d \times d}(B)$ equipped with the usual addition and the commutation rule

$$
\tau^{k} M=M^{(k)} \tau^{k}, \quad k \in \mathbb{N} .
$$


Now let $L$ be a field extension of $\mathbb{F}_{q}$ equipped with an $\mathbb{F}_{q}$-algebra homomorphism $\iota: A \rightarrow L$. An Anderson A-module (or an Anderson module for short) of dimension $d$ over $L$ is an $\mathbb{F}_{q^{-}}$-algebra homomorphism

$$
E: A \rightarrow M_{d \times d}(L)\{\tau\}
$$

such that

1) For all $a \in A$, if we write $E_{a}=\sum_{k \geq 0} E_{a, k} \tau^{k}, E_{a, k} \in M_{d \times d}(L)$, then we require

$$
\left(E_{a, 0}-\iota(a) I_{d}\right)^{d}=0_{d}
$$

2) There exists $a \in A$ such that $E_{a} \neq E_{a, 0}$.

We denote by $\partial_{E}: A \rightarrow M_{d \times d}(L)$ the $\mathbb{F}_{q}$-algebra homomorphism given by $\partial_{E}(a)=$ $E_{a, 0}$ for $a \in A$.

Remark 2.1. By definition, a Drinfeld module over $L$ is an Anderson module of dimension 1 over $L$, i.e. it is an $\mathbb{F}_{q}$-algebra homomorphism

$$
\phi: A \rightarrow L\{\tau\}
$$

such that

1) For all $a \in A$, if we write $\phi_{a}=\sum_{k \geq 0} \phi_{a, k} \tau^{k}, \phi_{a, k} \in L$, then we have $\phi_{a, 0}=\iota(a)$.

2) There exists $a \in A$ such that $\phi_{a} \neq \phi_{a, 0}$.

Let $E$ be an Anderson module of dimension $d$ over $L$ as above and let $B$ be an $A$-algebra. We can define two $A$-module structures on $B^{d}$. The first one is denoted by $E(B)$ where $A$ acts on $B^{d}$ via $E$ :

$$
a \cdot\left(\begin{array}{c}
m_{1} \\
\vdots \\
m_{d}
\end{array}\right)=\sum_{k \geq 0} E_{a, k}\left(\begin{array}{c}
m_{1}^{q^{k}} \\
\vdots \\
m_{d}^{q^{k}}
\end{array}\right), \quad \text { for } a \in A,\left(\begin{array}{c}
m_{1} \\
\vdots \\
m_{d}
\end{array}\right) \in B^{d} .
$$

The second one is denoted by $\operatorname{Lie}(E)(M)$ where $A$ acts on $B^{d}$ via $\partial_{E}$ :

$$
a \cdot\left(\begin{array}{c}
m_{1} \\
\vdots \\
m_{d}
\end{array}\right)=E_{a, 0}\left(\begin{array}{c}
m_{1} \\
\vdots \\
m_{d}
\end{array}\right), \quad \text { for } a \in A,\left(\begin{array}{c}
m_{1} \\
\vdots \\
m_{d}
\end{array}\right) \in B^{d} .
$$

\subsection{The Carlitz module.}

When $A=\mathbb{F}_{q}[\theta]$, i.e. the basic genus 0 case (see Example 1.1), the first example of a Drinfeld module is the Carlitz module over $K$ defined by Carlitz [19] in 1935. It is the $\mathbb{F}_{q}$-algebra homomorphism $C: A \rightarrow K\{\tau\}$ given by

$$
C_{\theta}=\theta+\tau \text {. }
$$

More generally, a Drinfeld module over $L$ is an $\mathbb{F}_{q}$-algebra homomorphism $\phi: A \rightarrow$ $L\{\tau\}$ given by

$$
\phi_{\theta}=\iota(\theta)+\text { higher terms in } \tau \text {. }
$$




\subsection{Tensor powers of the Carlitz module.}

We still work with $A=\mathbb{F}_{q}[\theta]$. Then an Anderson module of dimension $d$ over $L$ is an $\mathbb{F}_{q}$-algebra homomorphism $E: A \rightarrow M_{d \times d}(L)\{\tau\}$ given by a non-constant twisted polynomial

$$
E_{\theta}=\sum_{k \geq 0} E_{\theta, k} \tau^{k}, E_{\theta, k} \in M_{d \times d}(L)
$$

such that

$$
\left(E_{\theta, 0}-\iota(\theta) I_{d}\right)^{d}=0_{d}
$$

Of special interest are tensor powers of the Carlitz module which are studied in details by Anderson and Thakur [7]. Let $n \geq 1$ be an integer. We define the $n$-th tensor power of the Carlitz module $C^{\otimes n}: A \rightarrow M_{n \times n}(K)\{\tau\}$ by

$$
C_{\theta}^{\otimes n}=\left(\begin{array}{cccc}
\theta & 1 & \cdots & 0 \\
& \theta & \ddots & \vdots \\
& & \theta & 1 \\
& & & \theta
\end{array}\right)+\left(\begin{array}{cccc}
0 & 0 & \cdots & 0 \\
\vdots & \vdots & & \vdots \\
0 & 0 & \cdots & 0 \\
1 & 0 & \cdots & 0
\end{array}\right) \tau .
$$

\subsection{Finite Drinfeld modules.}

For general $A$, Gekeler [30] investigated finite Drinfeld modules, i.e. those defined over a finite field $L$. He developed an analogy of these modules with abelian varieties and obtained the analogue of Tate's isogeny theory.

\subsection{Sign-normalized rank one Drinfeld modules.}

For general $A$, in parallel with the seminal work of Drinfeld [25, 26], Hayes [37, 38] studied the theory of rank one Drinfeld modules which play the role of the Carlitz module when $A=\mathbb{F}_{q}[\theta]$. This is the reason why one often refers sign-normalized rank one Drinfeld modules to Drinfeld-Hayes modules.

For simplicity, we suppose that $d_{\infty}=1$. We refer the interested reader to Hayes' work [37, 38] (see also [3, 57] for $d_{\infty}=1$ and [10] or [31, Chapter 7 for general $\left.d_{\infty}\right)$. By definition, a sign-normalized rank one Drinfeld module is an $\mathbb{F}_{q}$-algebra homomorphism $\phi: A \rightarrow \bar{K}_{\infty}\{\tau\}$ such that

$$
\phi_{a}=a+\cdots+\operatorname{sgn}(a) \tau^{\operatorname{deg} a}, \quad \text { for } a \in A \backslash\{0\} .
$$

By [31, Theorem 7.2.15, there always exist sign-normalized rank one Drinfeld modules.

Let $H$ be the Hilbert class field of A, i.e. the maximal abelian everywhere unramified extension of $K$ in which $\infty$ splits completely. Denote by $O_{H}$ the integral closure of $A$ in $H$. One can show that $\phi_{a} \in O_{H}\{\tau\}$ for $a \in A$. Further, if $\phi$ is a sign-normalized rank one Drinfeld module and $\sigma \in \operatorname{Gal}(H / K)$, then $\sigma(\phi)$ is also a sign-normalized rank one Drinfeld module. This gives rise to the bijection between the set of sign-normalized rank one Drinfeld modules and the finite set $\operatorname{Gal}(H / K)$. We note that the cardinal of $\mathrm{Gal}(H / K)$ is equal to the class number $|\mathrm{Cl}(K)|$ of $K$. In particular, there are exactly $|\mathrm{Cl}(K)|$ sign-normalized rank one Drinfeld modules.

Example 2.2 (The basic genus 0 case). 
When $A=\mathbb{F}_{q}[\theta]$, the unique sign-normalized rank one Drinfeld module is the Carlitz module $C$.

Example 2.3 (Principal ideal domains).

We work with the four PIDs for which $\operatorname{Pic}(A)=1$ (see Example 1.2). Since $|\mathrm{Cl}(K)|=1$, there is a unique sign-normalized rank one Drinfeld module for each A. Thakur gave an explicit formula of these Drinfeld modules in [56, Sections II and III.

For example, in Example A where $A=\mathbb{F}_{3}[x, y] /\left(y^{2}-x^{3}+x+1\right)$, the unique sign-normalized rank one Drinfeld module is given by

$$
\begin{gathered}
\phi_{x}=x+x_{1} \tau+\tau^{2}, \\
\phi_{y}=y+y_{1} \tau+y_{2} \tau^{2}+\tau^{3},
\end{gathered}
$$

where

$$
x_{1}=y\left(x^{3}-x\right), \quad y_{1}=y\left(y^{3}-y\right), \quad y_{2}=y^{9}+y^{3}+y .
$$

Example 2.4 (Elliptic curves).

When $g=1$ and $d_{\infty}=1$, explicit formulas for sign-normalized rank one Drinfeld modules are given by Anderson ([2], Section 5.5). The interested reader could be referred to 34 for similar calculations.

\subsection{Tensor powers of sign-normalized rank one Drinfeld modules.}

One can define tensor powers of sign-normalized rank one Drinfeld modules by [36], Sections 2 and 5. These Anderson modules are studied in a forthcoming work of the authors 12. We should mention that in the case of elliptic curves when $g=1$ and $d_{\infty}=1$, they are investigated by a different method by Green [32, 33].

\section{Arithmetic of Anderson modules}

\subsection{Setup.}

We are now interested in Anderson modules defined over a finite extension of $K$. Let $L / K$ be a finite extension and let $O_{L}$ be the integral closure of $A$ in $L$. We set $L_{\infty}:=L \otimes_{K} K_{\infty}$.

Let $E / O_{F}$ be an Anderson module of dimension $d \geq 1$ defined over $O_{L}$, i.e. for all $a \in A$, we require that $E_{a} \in M_{d \times d}\left(O_{L}\right)\{\tau\}$. In particular, we have an $\mathbb{F}_{q}$-algebra homomorphism $\partial_{E}: A \rightarrow M_{d \times d}\left(O_{L}\right)$ which extends uniquely to a continuous map

$$
\partial_{E}: K_{\infty} \rightarrow M_{d \times d}\left(L_{\infty}\right) .
$$

\subsection{Exponential series and logarithm series.}

One can show that there exist unique power series $\exp _{E}, \log _{E} \in I_{d}+M_{d \times d}(L)\{\{\tau\}\} \tau$ satisfying the following equalities in $M_{d \times d}(L)\{\{\tau\}\}$ :

$$
\begin{aligned}
\exp _{E} \partial_{E}(a) & =E_{a} \exp _{E}, \quad a \in A, \\
\log _{E} E_{a} & =\partial_{E}(a) \log _{E}, \quad a \in A, \\
\exp _{E} \log _{E} & =\log _{E} \exp _{E}=I_{d} .
\end{aligned}
$$


These power series $\exp _{E}$ and $\log _{E}$ are called the exponential series and the logarithm series attached to $E$. The exponential series $\exp _{E}$ converges on $\operatorname{Lie}(E)\left(L_{\infty}\right)$ (see for example [31, Section 5.9, and the original article of G. Anderson [1]). In particular, $\exp _{E}$ induces a homomorphism of $A$-modules

$$
\exp _{E}: \operatorname{Lie}(E)\left(L_{\infty}\right) \rightarrow E\left(L_{\infty}\right) .
$$

We should stress that the logarithm series $\log _{E}$ does not converge everywhere but only on a neighborhood of 0 .

\subsection{Unit and class modules.}

Following L. Taelman [52], we introduce the unit module attached to $E / O_{L}$ as follows:

$$
U\left(E / O_{L}\right):=\left\{x \in \operatorname{Lie}(E)\left(L_{\infty}\right) \mid \exp _{E}(x) \in E\left(O_{L}\right)\right\} .
$$

By [52], one can easily deduce that $U\left(E / O_{L}\right)$ is in fact an $A$-lattice in $\operatorname{Lie}(E)\left(L_{\infty}\right)$. Further, the exponential series $\exp _{E}$ induces an exact sequence of $A$-modules:

$$
0 \rightarrow U\left(E / O_{L}\right) \rightarrow \operatorname{Lie}(E)\left(L_{\infty}\right) \rightarrow \frac{E\left(L_{\infty}\right)}{E\left(O_{L}\right)} \rightarrow \frac{E\left(L_{\infty}\right)}{E\left(O_{L}\right)+\exp _{E}\left(\operatorname{Lie}(E)\left(L_{\infty}\right)\right)} \rightarrow 0
$$

One can show that

$$
H\left(E / O_{L}\right):=\frac{E\left(L_{\infty}\right)}{E\left(O_{L}\right)+\exp _{E}\left(\operatorname{Lie}(E)\left(L_{\infty}\right)\right)}
$$

is a finite $A$-module which is called the class module attached to $E / O_{L}$.

If $M$ is an $A$-lattice in $\operatorname{Lie}(E)\left(L_{\infty}\right)$, the regulator of $M$ is defined by

$$
\operatorname{Reg}_{E / O_{L}}(M):=\left[\operatorname{Lie}(E)\left(O_{L}\right): M\right]_{A} .
$$

In particular,

$$
\operatorname{Reg}_{E / O_{L}}\left(U\left(E / O_{L}\right)\right):=\left[\operatorname{Lie}(E)\left(O_{L}\right): U\left(E / O_{L}\right)\right]_{A} .
$$

\subsection{Stark units.}

We recall the notion of Stark units which is first introduced by two of the authors for Drinfeld modules when $A=\mathbb{F}_{q}[\theta]$ ([16]) and subsequently developed by the authors for Anderson modules for general $A([10,11])$.

Let $z$ be an indeterminate over $K_{\infty}$ and let $\mathbb{T}_{z}\left(K_{\infty}\right):=\mathbb{F}_{\infty}[z]((\pi))$ be the Tate algebra in the variable $z$ with coefficients in $K_{\infty}$. We set

$$
\mathbb{T}_{z}\left(L_{\infty}\right):=L_{\infty} \otimes_{K_{\infty}} \mathbb{T}_{z}\left(K_{\infty}\right) .
$$

The map $\tau: L_{\infty} \rightarrow L_{\infty}, x \mapsto x^{q}$, extends uniquely into a continuous homomorphism of $\mathbb{F}_{q}[z]$-algebras $\tau: \mathbb{T}_{z}\left(L_{\infty}\right) \rightarrow \mathbb{T}_{z}\left(L_{\infty}\right)$. Let $\widetilde{E}: A[z] \rightarrow M_{d \times d}(L[z])\{\tau\}$ be the morphism of $\mathbb{F}_{q}[z]$-algebras such that

$$
\widetilde{E}_{a}=\sum_{k \geq 0} z^{k} E_{a, k} \tau^{k}, \quad a \in A .
$$

There exists a unique element $\exp _{\widetilde{E}} \in I_{d}+\tau M_{d \times d}(L[z])\{\{\tau\}\}$ such that

$$
\exp _{\widetilde{E}} \partial_{E}(a)=\widetilde{E}_{a} \exp _{\widetilde{E}}, \quad a \in A .
$$


If $\exp _{E}=\sum_{i \geq 0} E_{i} \tau^{i}, E_{i} \in M_{d \times d}(L)$, one can show that

$$
\exp _{\widetilde{E}}=\sum_{i \geq 0} z^{i} E_{i} \tau^{i}
$$

In particular, $\exp _{\widetilde{E}}$ converges on $\operatorname{Lie}(\widetilde{E})\left(\mathbb{T}_{z}\left(L_{\infty}\right)\right)$ and induces a homomorphism of $A[z]$-modules

$$
\exp _{\widetilde{E}}: \operatorname{Lie}(\widetilde{E})\left(\mathbb{T}_{z}\left(L_{\infty}\right)\right) \rightarrow \widetilde{E}\left(\mathbb{T}_{z}\left(L_{\infty}\right)\right)
$$

Let ev $: \operatorname{Lie}(\widetilde{E})\left(\mathbb{T}_{z}\left(L_{\infty}\right)\right) \rightarrow \operatorname{Lie}(E)\left(L_{\infty}\right)$ be the evaluation at $z=1$. Observe that ev induces a short exact sequence of $A$-modules :

$$
0 \rightarrow(z-1) \operatorname{Lie}(\widetilde{E})\left(\mathbb{T}_{z}\left(L_{\infty}\right)\right) \rightarrow \operatorname{Lie}(\widetilde{E})\left(\mathbb{T}_{z}\left(L_{\infty}\right)\right) \rightarrow \operatorname{Lie}(E)\left(L_{\infty}\right) \rightarrow 0 .
$$

We define

$$
U_{S t}\left(E / O_{L}\right):=\operatorname{ev}\left(\left\{x \in \operatorname{Lie}(\widetilde{E})\left(\mathbb{T}_{z}\left(L_{\infty}\right)\right) \mid \exp _{\widetilde{E}}(x) \in \widetilde{E}\left(O_{L}[z]\right)\right\}\right) .
$$

We observe that $U_{S t}\left(E / O_{L}\right) \subset U\left(E / O_{L}\right)$. The $A$-module $U_{S t}\left(E / O_{L}\right)$ is called the module of Stark units attached to $E / O_{L}$. One can prove that $U_{S t}\left(E / O_{L}\right)$ is an $A$-lattice in $\operatorname{Lie}(E)\left(L_{\infty}\right)$ and that we have (see [10, 11, 16]):

$$
\left[\frac{U\left(E / O_{L}\right)}{U_{S t}\left(E / O_{L}\right)}\right]_{A}=\left[H\left(E / O_{L}\right)\right]_{A} \text {. }
$$

In particular,

$$
\operatorname{Reg}_{E / O_{L}}\left(U_{S t}\left(E / O_{L}\right)\right)=\operatorname{Reg}_{E / O_{L}}\left(U\left(E / O_{L}\right)\right) \cdot\left[H\left(E / O_{L}\right)\right]_{A} .
$$

We observe that $\left[H\left(E / O_{L}\right)\right]_{A} \in \bar{K}$ where $\bar{K}$ is the algebraic closure of $K$ in $\bar{K}_{\infty}$. This leads us to the following open problem:

Problem 3.1. Let $E / O_{L}$ be an Anderson module. Show that

$$
\operatorname{Reg}_{E / O_{L}}\left(U_{S t}\left(E / O_{L}\right)\right) \notin \bar{K} \text {. }
$$

\section{The Class formula À la Taelman}

We continue with the notation of Section 3.1. Recall that $L / K$ is a finite extension and $O_{L}$ is the integral closure of $A$ in $L$. We have set $L_{\infty}=L \otimes_{K} K_{\infty}$.

\subsection{The class formula à la Taelman: a conjecture.}

Let $E$ be an Anderson module of dimension $d$ defined over $O_{L}$. For any maximal ideal $\mathfrak{P}$ of $O_{L}$, we put $\mathbb{F}_{\mathfrak{P}}=\frac{O_{L}}{\mathfrak{P}}$ which is a finite extension of $\mathbb{F}_{q}$. Then $E\left(\mathbb{F}_{\mathfrak{P}}\right)$ and $\operatorname{Lie}(E)\left(\mathbb{F}_{\mathfrak{P}}\right)$ are finite $A$-modules. Thus it makes sense to consider the quotient $\frac{\left[\operatorname{Lie}(E)\left(\mathbb{F}_{\mathfrak{P}}\right)\right]_{A}}{\left[E\left(\mathbb{F}_{\mathfrak{P}}\right)\right]_{A}}$. The special $L$-value attached to $E / O_{L}$ is defined by

$$
L_{A}\left(E / O_{L}\right):=\prod_{\mathfrak{P}} \frac{\left[\operatorname{Lie}(E)\left(\mathbb{F}_{\mathfrak{P}}\right)\right]_{A}}{\left[E\left(\mathbb{F}_{\mathfrak{P}}\right)\right]_{A}},
$$

where $\mathfrak{P}$ runs through the maximal ideals of $O_{L}$.

We state a conjecture on special $L$-values of Anderson modules which is also known as the class formula à la Taelman: 
Conjecture 4.1. Let $E$ be an Anderson module defined over $O_{L}$. Then

1) (Convergence) The infinite product

$$
L_{A}\left(E / O_{L}\right)=\prod_{\mathfrak{P}} \frac{\left[\operatorname{Lie}(E)\left(\mathbb{F}_{\mathfrak{P}}\right)\right]_{A}}{\left[E\left(\mathbb{F}_{\mathfrak{P}}\right)\right]_{A}}
$$

converges in $\mathbb{C}_{\infty}$.

2) (Class formula) We have the class formula for $E / O_{L}$ :

$$
\operatorname{Reg}_{E / O_{L}}\left(U_{S t}\left(E / O_{L}\right)\right)=L_{A}\left(E / O_{L}\right),
$$

or equivalently,

$$
\left[\operatorname{Lie}(E)\left(O_{L}\right): U\left(E / O_{L}\right)\right]_{A} \cdot\left[H\left(E / O_{L}\right)\right]_{A}=L_{A}\left(E / O_{L}\right) .
$$

We mention below what is known about this Conjecture.

(1) When $A=\mathbb{F}_{q}[\theta]$ (see Example 1.1), Conjecture 4.1 is true for the Carlitz module defined over the ring $A$ itself by the pioneer work of Carlitz [19]. In 2012, Taelman made a breakthrough and proved that Conjecture 4.1 holds for any Drinfeld module $E / O_{L}$. Shortly after, Fang [29] and Demeslay [23, 24, proved the class formula for any Anderson module $E / O_{L}$. While the proof of Demeslay follows closely the original proof of Taelman, that of Fang is based on the work of V. Lafforgue 41] using shtukas and the theory of Fontaine in equal characteristics. In particular, the convergence part relies heavily on the works of Anderson [4] and Böckle and Pink ([17, Chapter 8).

(2) For the four extra rings $A$ which are PIDs (see Example 1.2), Thakur [56, 59. proved the class formula for the unique sign-normalized rank one Drinfeld module over $A$ (see Example 2.3).

(3) For general $A$, in [10, the authors succeeded in proving the above Conjecture for sign-normalized rank one Drinfeld modules over any finite extension of $K$. The proof is based on the work of Gekeler [30] for the convergence and on the arithmetic of Stark units for the class formula.

(4) For general $A$ satisfying the hypothesis that $p$ does not divide $|\mathrm{Cl}(K)|$, Debry 22] showed that Conjecture 4.1 holds for any Drinfeld module $E / O_{L}$.

(5) For general $A$, the validity of this Conjecture for Drinfeld modules having good reduction everywhere is a consequence of the recent work of M. Mornev [45].

(6) For general $A$, Taelman ([51, Proposition 8) showed that if $E$ is abelian or $A$-finite (see [36], Section 5 for definitions), then the convergence part (Conjecture 4.1. Part 1)) holds. In particular, $L_{A}\left(E / O_{L}\right)$ is well-defined for any Drinfeld module $E / O_{F}$ since any Drinfeld module is abelian and $A$-finite (see for example [36, Corollary 5.15).

In the rest of this Section, based on the work of Debry [22], we will explain the validity of this Conjecture for any Anderson module $E / O_{L}$ under the hypothesis that $p$ does not divide $|\mathrm{Cl}(K)|$, see Theorem 4.4 .

\subsection{A theorem of Debry and its application to the class formula.}


We set

$$
Z(K, \infty):=\left\{x \in 1+\pi \mathbb{F}_{\infty}[[\pi]] \mid N_{K_{\infty} / \mathbb{F}_{q}\left(\left(\frac{1}{a}\right)\right)}(x)=1 \text { for all } a \in A \backslash \mathbb{F}_{q},\right\} .
$$

We note that $1+\pi \mathbb{F}_{\infty}[[\pi]]$ is a $\mathbb{Z}_{p}$-module by exponentiation and that $Z(K, \infty)$ is a sub- $\mathbb{Z}_{p}$-module of $1+\pi \mathbb{F}_{\infty}[[\pi]]$. Recall that $\mathrm{Cl}(K)$ is the group of classes of degree zero divisor of $K$ and denote by $\mathrm{Cl}(K)[p]$ the $p$-torsion subgroup of $\mathrm{Cl}(K)$. By 22], Section 4 (see in particular Proposition 4.14, Corollary 4.15, Proposition 4.20, Corollary 4.21 and the Remarks after Corollary 4.22), it follows that $Z(K, \infty)$ is a finitely generated $\mathbb{Z}_{p}$-module. Further, Debry proved the following theorem ([22], Chapter 4):

Theorem 4.2 (Debry 2016). There exists an injective group homomorphism

$$
\frac{Z(K, \infty)}{Z(K, \infty)^{p}} \hookrightarrow \mathrm{Cl}(K)[p] .
$$

As an immediate application, we get the following corollary:

Corollary 4.3. Suppose that $p$ does not divide $|\mathrm{Cl}(K)|$. Let $\left(\alpha_{n}\right)_{n \in \mathbb{N}}$ be a sequence of elements in $K_{\infty}^{\times}$such that for all $n \in \mathbb{N}$, we have $\operatorname{sgn}\left(\alpha_{n}\right)=1$. We assume that for all $a \in A \backslash \mathbb{F}_{q}$ the infinite product $\prod_{n \in \mathbb{N}} N_{K_{\infty} / \mathbb{F}_{q}\left(\left(\frac{1}{a}\right)\right)}\left(\alpha_{n}\right)$ converges in $\mathbb{F}_{q}\left(\left(\frac{1}{a}\right)\right)$. Then the infinite product $\prod_{n \in \mathbb{N}} \alpha_{n}$ converges in $K_{\infty}^{\times}$.

Proof.

1) Since $p$ does not divide $|\mathrm{Cl}(K)|$, Theorem 4.2 implies that

$$
\frac{Z(K, \infty)}{Z(K, \infty)^{p}}=1
$$

Since $Z(K, \infty)$ is a finitely generated $\mathbb{Z}_{p}$-module, by Nakayama's Lemma, we obtain

$$
Z(K, \infty)=\{1\}
$$

2) We observe that

$$
\lim _{n \rightarrow+\infty} N_{K_{\infty} / \mathbb{F}_{q}\left(\left(\frac{1}{a}\right)\right)}\left(\alpha_{n}\right)=1, \quad \text { for all } a \in A \backslash \mathbb{F}_{q} .
$$

Since $\operatorname{sgn}\left(\alpha_{n}\right)=1$, we deduce that

$$
\alpha_{n} \in 1+\pi \mathbb{F}_{\infty}[[\pi]], \quad \text { for } n \gg 0 .
$$

3) Let $\left(\beta_{n}\right)_{n \in \mathbb{N}}$ be a convergent sub-sequence of $\left(\alpha_{n}\right)_{n \in \mathbb{N}}$. We put $\beta=\lim _{n \rightarrow+\infty} \beta_{n}$. It follows that

$$
N_{K_{\infty} / \mathbb{F}_{q}\left(\left(\frac{1}{a}\right)\right)}(\beta)=1, \quad \text { for all } a \in A \backslash \mathbb{F}_{q} .
$$

Thus $\beta \in Z(K, \infty)$. We conclude that $\beta=1$.

4) By Step 3), the sequence $\left(\alpha_{n}\right)_{n \in \mathbb{N}}$ admits only 1 as an accumulation point. Since $1+\pi \mathbb{F}_{\infty}[[\pi]]$ is compact, $\left(\alpha_{n}\right)_{n \in \mathbb{N}}$ converges to 1 . Hence $\prod_{n \in \mathbb{N}} \alpha_{n}$ converges in $K_{\infty}$. The proof is finished.

We are now ready to prove the main theorem of this Section. 
Theorem 4.4. Suppose that $p$ does not divide $|\mathrm{Cl}(K)|$. Let $E$ be an Anderson module defined over $O_{L}$. Then Conjecture 4.1 holds for $E / O_{L}$, i.e. $L_{A}\left(E / O_{L}\right)$ is well-defined and we have the equality:

$$
\operatorname{Reg}_{E / O_{L}}\left(U_{S t}\left(E / O_{L}\right)\right)=L_{A}\left(E / O_{L}\right) .
$$

Proof. Let $p^{u}$ be the biggest power of $p$ dividing $d_{\infty}$.

1) Since $p$ does not divide $|\mathrm{Cl}(K)|$, by the proof of Corollary 4.3 , it follows that

$$
Z(K, \infty)=1 \text {. }
$$

2) We claim that $L_{A}\left(E / O_{L}\right)$ converges. In fact, for any element $a \in A \backslash \mathbb{F}_{q}$, the Anderson module $E: A \rightarrow M_{d \times d}\left(O_{L}\right)\{\tau\}$ induces an Anderson module $E: \mathbb{F}_{q}[a] \rightarrow$ $M_{d \times d}\left(O_{L}\right)\{\tau\}$. We have already mentionned that $L_{\mathbb{F}_{q}[a]}\left(E / O_{L}\right)$ is well-defined by Demeslay [23, 24] and Fang [29]. (see the discussion after Conjecture 4.1).

Let $M$ be a finite $A$-module and let $\mathcal{N}_{K / \mathbb{F}_{q}(a)}: I(A) \rightarrow I\left(\mathbb{F}_{q}[a]\right)$ be the norm map on fractional ideals. Then for any finite $A$-module $M$, if Fitt $_{A} M$ denotes the Fitting ideal of $M$, we have

$$
\mathcal{N}_{K / \mathbb{F}_{q}(a)}\left(\operatorname{Fitt}_{A} M\right)=\operatorname{Fitt}_{\mathbb{F}_{q}[a]} M .
$$

It follows that

$$
\prod_{\mathfrak{P}} N_{K_{\infty} / \mathbb{F}_{q}\left(\left(\frac{1}{a}\right)\right)}\left(\left(\frac{\left[\operatorname{Lie}(E)\left(\mathbb{F}_{\mathfrak{P}}\right)\right]_{A}}{\left[E\left(\mathbb{F}_{\mathfrak{P}}\right)\right]_{A}}\right)^{p^{u}\left(q^{d_{\infty}}-1\right)}\right)=L_{\mathbb{F}_{q}[a]}\left(E / O_{L}\right)^{p^{u}\left(q^{d \infty}-1\right)} .
$$

We observe that for any maximal ideal $\mathfrak{P}$ of $O_{L}$, we have

$$
\operatorname{sgn}\left(\left(\frac{\left[\operatorname{Lie}(E)\left(\mathbb{F}_{\mathfrak{P}}\right)\right]_{A}}{\left[E\left(\mathbb{F}_{\mathfrak{P}}\right)\right]_{A}}\right)^{p^{u}}\right)=1 .
$$

By Corollary 4.3 we conclude that $L_{A}\left(E / O_{L}\right)$ is well-defined.

3) Now we prove the class formula for $E / O_{L}$. We observe that $L_{A}\left(E / O_{L}\right)^{p^{u}}$ and $\operatorname{Reg}_{E / O_{L}}\left(U_{S t}\left(E / O_{L}\right)\right)^{p^{u}}$ lie in $K_{\infty}$. Thus

$$
N_{K_{\infty} / \mathbb{F}_{q}\left(\left(\frac{1}{a}\right)\right)}\left(L_{A}\left(E / O_{L}\right)^{p^{u}}\right)^{q^{d \infty}-1}=L_{\mathbb{F}_{q}[a]}\left(E / O_{L}\right)^{p^{u}\left(q^{d \infty}-1\right)},
$$

and

$$
N_{K_{\infty} / \mathbb{F}_{q}\left(\left(\frac{1}{a}\right)\right)}\left(\operatorname{Reg}_{E / O_{L}}\left(U_{S t}\left(E / O_{L}\right)\right)^{p^{u}}\right)^{q^{d \infty}-1}=\operatorname{Reg}_{E / O_{L}}^{\mathbb{F}_{q}[a]}\left(U_{S t}\left(E / O_{L}\right)\right)^{p^{u}\left(q^{d \infty}-1\right)},
$$

where $\operatorname{Reg}_{E / O_{L}}^{\mathbb{F}_{q}[a]}$ denotes the regulator of the induced Anderson module $E: \mathbb{F}_{q}[a] \rightarrow$ $M_{d \times d}\left(O_{L}\right)\{\tau\}$.

By the class formula for the induced Anderson modules $E: \mathbb{F}_{q}[a] \rightarrow M_{d \times d}\left(O_{L}\right)\{\tau\}$ for $a \in A \backslash \mathbb{F}_{q}$ (see [23, 24, 29]), we obtain

$$
\left(\frac{L_{A}\left(E / O_{L}\right)}{\operatorname{Reg}_{E / O_{L}}\left(U_{S t}\left(E / O_{L}\right)\right)}\right)^{p^{u}} \in \mathbb{F}_{\infty}^{\times} Z(K, \infty) .
$$

We note that

$$
\operatorname{sgn}\left(L_{A}\left(E / O_{L}\right)^{p^{u}}\right)=\operatorname{sgn}\left(\operatorname{Reg}_{E / O_{L}}\left(U_{S t}\left(E / O_{L}\right)^{p^{u}}\right)=1 .\right.
$$


Thus

$$
\left(\frac{L_{A}\left(E / O_{L}\right)}{\operatorname{Reg}_{E / O_{L}}\left(U_{S t}\left(E / O_{L}\right)\right)}\right)^{p^{u}} \in Z(K, \infty) .
$$

Since $Z(K, \infty)=1$, we obtain

$$
\left(\frac{L_{A}\left(E / O_{L}\right)}{\operatorname{Reg}_{E / O_{L}}\left(U_{S t}\left(E / O_{L}\right)\right)}\right)^{p^{u}}=1 .
$$

The class formula follows.

The proof of Theorem 4.4 is finished.

To end this Section, we would like to ask the following question.

Problem 4.5. Do there exist a global function field $K / \mathbb{F}_{q}$ and a place $\infty$ of $K$ such that $Z(K, \infty) \neq\{1\}$ ?

\section{An example: tensor powers of the Carlitz module}

In this section, we work with tensor powers $C^{\otimes n}$ of the Carlitz module for $n \in \mathbb{N}^{*}$ which are studied extensively by Anderson and Thakur in 7. We report our recent work 11] which explains how to derive log-algebraicity identities from the class formula for $C^{\otimes n}$.

Throughout this section, we deal with the basic genus 0 case (see Example 1.1). Recall that $K=\mathbb{F}_{q}(\theta), A=\mathbb{F}_{q}[\theta]$ and $K_{\infty}=\mathbb{F}_{q}\left(\left(\frac{1}{\theta}\right)\right)$ equipped with the discrete valuation $v_{\infty}$. For $d \in \mathbb{N}$, recall that $A_{+, d}$ denotes the set of monic elements in $A$ of degree $d$.

\subsection{The Carlitz-Goss zeta function and its special values.}

We briefly recall the definition and some properties of the Carlitz-Goss zeta function. We refer the interested reader to 31, Chapter 8 for more details.

Let $\mathbb{S}_{\infty}=\mathbb{C}_{\infty}^{\times} \times \mathbb{Z}_{p}$. We have an injective group homomorphism

$$
\begin{aligned}
s: \mathbb{Z} & \rightarrow \mathbb{S}_{\infty} \\
n & \mapsto\left(\theta^{n}, n\right) .
\end{aligned}
$$

For $(x, y) \in \mathbb{S}_{\infty}$, the following infinite sum converges in $\mathbb{C}_{\infty}$ :

$$
\zeta_{A}((x, y))=\sum_{d \geq 0} \sum_{a \in A_{+, d}}\left(\frac{a}{\theta^{d}}\right)^{-y} x^{-d} .
$$

The function $\zeta_{A}: \mathbb{S}_{\infty} \rightarrow \mathbb{C}_{\infty}$ is called the Carlitz-Goss zeta function. The Carlitz zeta values are the special values of this zeta function and are given by

$$
\zeta_{A}(n):=\zeta_{A}(s(n))=\sum_{d \geq 0} \sum_{a \in A_{+, d}} a^{-n}, \quad n \in \mathbb{Z}
$$


One can prove that $\zeta_{A}(0)=1$ and one sees that $\zeta_{A}(n)$ converges in $K_{\infty}$ for $n>0$. The Carlitz-Goss zeta function has trivial zeroes: for $n<0$ and $n \equiv 0(\bmod q-1)$, we have

$$
\zeta_{A}(n)=0 .
$$

Furthermore, for $n<0$ and $n \not \equiv 0(\bmod q-1)$, one can show that

$$
\zeta_{A}(n) \in A \backslash\{0\} \text {. }
$$

Recall (see Example 2.2) that $C / A$ is the Carlitz module over $A$ given by

$$
C_{\theta}=\theta+\tau \text {. }
$$

Then there exists $\tilde{\pi} \in \bar{K}_{\infty}$ called the Carlitz period such that

$$
\left\{x \in \mathbb{C}_{\infty}, \exp _{C}(x)=0\right\}=\tilde{\pi} A \text {. }
$$

One can prove that

$$
\widetilde{\pi}=(-\theta)^{\frac{1}{q-1}} \theta \prod_{k \geq 1}\left(1-\frac{\theta}{\theta^{q^{k}}}\right)^{-1}
$$

In 1941, Wade [60] showed that $\tilde{\pi} \notin \bar{K}$, i.e. $\tilde{\pi}$ is transcendental over $K$. Furthermore, for $n \geq 1$ such that $n \equiv 0(\bmod q-1)$, we have

$$
\frac{\zeta_{A}(n)}{\widetilde{\pi}^{n}} \in K^{\times} \text {. }
$$

In 1991, J. Yu proved that $\zeta_{A}(n) \notin \bar{K}$ for $n \in \mathbb{N}^{*}$ and furthermore, for all $n \geq 1$ such that $n \not \equiv 0(\bmod q-1), \frac{\zeta_{A}(n)}{\widetilde{\pi}^{n}} \notin \bar{K}$.

\section{Problem 5.1.}

Does the Carlitz-Goss zeta function satisfies a kind of functional equation reminiscent that of the Riemann zeta function?

\subsection{The class formula implies log-algebricity identities.}

This section is based on the pioneer work of Anderson-Thakur [7] (see also [14, 46]) and on the recent paper of the authors ([11, Section 5). The reader interested by similar results for general $A$ could read [2, 3, 10].

Let $n \geq 1$ be an integer and let $k_{0}$ be the smallest integer such that $q^{k_{0}} \geq n$. Recall (see Example 2.3 that the $n$-th tensor power of the Carlitz module $C^{\otimes n}$ : $A \rightarrow M_{n \times n}(A)\{\tau\}$ is the Anderson module given by

$$
C_{\theta}^{\otimes n}=\left(\begin{array}{cccc}
\theta & 1 & \cdots & 0 \\
& \theta & \ddots & \vdots \\
& & \theta & 1 \\
& & & \theta
\end{array}\right)+\left(\begin{array}{cccc}
0 & 0 & \cdots & 0 \\
\vdots & \vdots & & \vdots \\
0 & 0 & \cdots & 0 \\
1 & 0 & \cdots & 0
\end{array}\right) \tau .
$$

The map $\partial_{C \otimes n}: A \rightarrow M_{n \times n}(A)$ is given by

$$
\partial_{C \otimes n}(\theta)=\left(\begin{array}{cccc}
\theta & 1 & \cdots & 0 \\
& \theta & \ddots & \vdots \\
& & \theta & 1 \\
& & & \theta
\end{array}\right) .
$$


We note that

$$
L\left(C^{\otimes n} / A\right)=\zeta_{A}(n) .
$$

In 1990, Anderson and Thakur proved the following fundamental result (7], Theorem 3.8.3): for $n \geq 1$, there exists $z_{n} \in \operatorname{Lie}\left(C^{\otimes n}\right)\left(K_{\infty}\right)$ such that $\exp _{C \otimes n}\left(z_{n}\right) \in$ $C^{\otimes n}(A)$ and

$$
\Gamma_{n} \zeta_{A}(n)=\iota\left(z_{n}\right),
$$

where $\exp _{C \otimes n}$ is the exponential series associated to $C^{\otimes n}, \iota$ is the projection onto the last coordinate and $\Gamma_{n} \in A$ is the Carlitz factorial (see [31], Section 9). The proof of Anderson and Thakur is based on the explicit expression of power sums and the Anderson-Thakur polynomials.

Recall that the logarithm series $\log _{C \otimes n} \in I_{n}+\tau M_{n \times n}(K)\{\{\tau\}\}$ satisfies

$$
\log _{C \otimes n} \exp _{C \otimes n}=\exp _{C \otimes n} \log _{C \otimes n}=I_{n} .
$$

We put $\log _{C \otimes n}=\sum_{k \geq 0} L_{k} \tau^{k}, L_{k} \in M_{n \times n}(K)$. We denote by $\iota: \operatorname{Lie}\left(C^{\otimes n}\right)\left(K_{\infty}\right) \rightarrow$ $K_{\infty}$ the projection on the last coordinate. Finally, let $W \subset \operatorname{Lie}\left(C^{\otimes n}\right)\left(K_{\infty}\right)$ be the $K_{\infty}$-vector space generated by $L_{k_{0}} \tau^{k}(x), x \in \operatorname{Lie}\left(C^{\otimes n}\right)\left(K_{\infty}\right)$. In [11], we prove the following properties:

1) $\operatorname{dim}_{K_{\infty}} W=1$ and $\iota(W) \neq\{0\}$.

2) $U_{S t}\left(C^{\otimes n} / A\right) \subset \operatorname{Lie}\left(C^{\otimes n}\right)(K)+W$.

Recall that the class formula for $C^{\otimes n} / A$ states that

$$
\operatorname{Reg}_{C \otimes n} / A\left(U_{S t}\left(C^{\otimes n} / A\right)\right)=\zeta_{A}(n) .
$$

Combining with the above properties, it follows that there exists an explicit element $x_{n} \in \operatorname{Lie}\left(C^{\otimes n}\right)(K) \cap W$ such that $\iota\left(x_{n}\right) \in K^{\times}$and

$$
\partial_{C \otimes n}(A)\left(\partial_{C \otimes n}\left(\zeta_{A}(n)\right) x_{n}\right)=U_{S t}\left(C^{\otimes n} / A\right) \cap W .
$$

We note that $U_{S t}\left(C^{\otimes n} / A\right) \cap W$ is an $A$-lattice in $W$. In particular, by projecting on the last coordinate, we rediscover the above theorem of Anderson-Thakur:

$$
\zeta_{A}(n) \iota\left(x_{n}\right) A \subset \iota\left(U_{S t}\left(C^{\otimes n} / A\right)\right) .
$$

\section{Remark 5.2.}

1) By similar arguments, one can obtain a log-algebraicity identity with an extra variable $z$ (see [11, Theorem 5.3).

2) We should mention that in a work in progress, Papanikolas 46 obtains similar log-algebraicity identities for $C^{\otimes n}$. His proof is based on Anderson's method developed in [2, 3].

\section{Further READING ON RELATED TOPICS}

In the last section, apart from the excellent books of Goss [31] and Thakur [58, we would like to give a list of references for related topics on Anderson modules. We would stress that this list is far from exhaustive. 


\subsection{Anderson motives and dual motives.}

In the same paper [1, Anderson gave the definition of Anderson motives which is closely related to that of Anderson modules. Later, he [5] introduced the notion of Anderson dual motives which turns out to be useful for transcendence theory.

- For definitions and basic properties, we refer to the seminal paper of Anderson [1] and also [5, 18] and [31, Section 5 for $A=\mathbb{F}_{q}[\theta]$. For general $A$, one can read the excellent survey of Hartl and Juschka [36].

- For the Tannakian theory of motives, we refer to the papers of Papanikolas [47] and Taelman 50.

- For Pink's theory of Hodge structures over function fields, one could also read [36].

\subsection{Shtukas.}

In his seminal papers 27, 28 on Langlands correspondence for GL(2) over function fields, Drinfeld extended the notion of Drinfeld modules and introduced the notion of shtukas, which plays a dominant role in the Langlands program over function fields (see [39, 40, 42, 43] for recent developments).

It turns out that shtukas and its variants such as $\tau$-sheaves and local shtukas are also very useful for arithmetic questions of Anderson modules. We refer the interested reader to 31, Chapter 6 and [10, 35, 41, 44, 45, 55, 57] for further information.

\subsection{Special $L$-values of Anderson modules.}

Special $L$-values of the Carlitz module over a cyclotomic extension are studied in $[15,53]$.

For $A=\mathbb{F}_{q}[\theta]$, deformations of special $L$-values of the Carlitz-Goss zeta function in Tate algebras are studied by Pellarin and two of the authors [13, 14, 16, 48, (see also [8] for arithmetic applications). For general $A$, one could read recent developments in 9 , 10, 34].

We should mention a conjecture of Taelman on special $L$-values of Anderson modules. It was first formulated by Taelman in [51] and studied extensively in [11.

\subsection{Log-algebraicity.}

To our knowledge, little is known about log-algebraicity. The most important results are due to Anderson who extended examples of Carlitz [19] and Thakur [56] and proved several identities for signed-normalized rank one Drinfeld modules. These results are extended and revisited by $[10,13,14,16,46]$ and [58, Chapter 8 .

\subsection{Transcendence theory.}

For applications to transcendence questions of special $L$-values of Anderson modules when $A=\mathbb{F}_{q}[\theta]$, we refer the reader to [6, 21, 49, 61, 62] or to a recent survey of Chang 20]. 


\section{REFERENCES}

[1] G. Anderson. $t$-motives. Duke Math. J., 53(2):457-502, 1986.

[2] G. Anderson. Rank one elliptic A-modules and A-harmonic series. Duke Math. J., 80(2):291303, 1994.

[3] G. Anderson. Log-algebraicity of twisted $A$-harmonic series and special values of $L$-series in characteristic $p$. J. Number Theory, 60(1):165-209, 1996.

[4] G. Anderson. An elementary approach to L-functions mod p. J. Number Theory, 73(3):491$542,2000$.

[5] G. Anderson, D. Brownawell, and M. Papanikolas. Rorhlich Conjecture Project. unpublished notes, 2002.

[6] G. Anderson, D. Brownawell, and M. Papanikolas. Determination of the algebraic relations among special $\Gamma$-values in positive characteristic. Ann. of Math. (2), 160:237-313, 2004.

[7] G. Anderson and D. Thakur. Tensor powers of the Carlitz module and zeta values. Ann. of Math. (2), 132(1):159-191, 1990.

[8] B. Anglès, T. Ngo Dac, and F. Tavares Ribeiro. Exceptional zeros of $L$-series and BernoulliCarlitz numbers. arXiv:1511.06209.

[9] B. Anglès, T. Ngo Dac, and F. Tavares Ribeiro. Special functions and twisted $L$-series. J. Théor. Nombres Bordeaux, 29:931-961, 2017.

[10] B. Anglès, T. Ngo Dac, and F. Tavares Ribeiro. Stark units in positive characteristic. Proc. Lond. Math. Soc. (3), 115(4):763-812, 2017.

[11] B. Anglès, T. Ngo Dac, and F. Tavares Ribeiro. On special $L$-values of $t$-modules. available at https://hal.archives-ouvertes.fr/hal-01901571, 2018.

[12] B. Anglès, T. Ngo Dac, and F. Tavares Ribeiro. Tensor powers of sign-normalized rank one Drinfeld modules. in preparation, 2018.

[13] B. Anglès, F. Pellarin, and F. Tavares Ribeiro. Arithmetic of positive characteristic $L$-series values in Tate algebras. With an appendix by F. Demeslay. Compos. Math., 152(1):1-61, 2016.

[14] B. Anglès, F. Pellarin, and F. Tavares Ribeiro. Anderson-Stark units for $\mathbb{F}_{q}[\theta]$. Trans. Amer. Math. Soc., 370(3):1603-1627, 2018.

[15] B. Anglès and L. Taelman. Arithmetic of characteristic $p$ special $L$-values. With an appendix by V. Bosser. Proc. Lond. Math. Soc. (3), 110(4):1000-1032, 2015.

[16] B. Anglès and F. Tavares Ribeiro. Arithmetic of function fields units. Math. Ann., 367(12):501-579, 2017.

[17] G. Böckle and R. Pink. Cohomological theory of crystals over function fields, volume 9 of EMS Tracts in Mathematics. European Mathematical Society (EMS), Zürich, 2009.

[18] D. Brownawell and M. Papanikolas. A rapid introduction to Drinfeld modules, $t$-modules and $t$-motives. In G. Böckle, D. Goss, U. Hartl, and M. Papanikolas, editors, Proceedings of the conference on "t-motives: Hodge structures, transcendence and other motivic aspects", BIRS, Banff, Canada 2009. European Mathematical Society, 2016.

[19] L. Carlitz. On certain functions connected with polynomials in Galois field. Duke Math. J., 1(2):137-168, 1935.

[20] C.Y. Chang. Frobenius difference equations and difference Galois groups. In G. Böckle, D. Goss, U. Hartl, and M. Papanikolas, editors, Proceedings of the conference on "t-motives: Hodge structures, transcendence and other motivic aspects", BIRS, Banff, Canada 2009. European Mathematical Society, 2016.

[21] C.Y. Chang and M. Papanikolas. Algebraic independence of periods and logarithms of Drinfeld modules. J. Amer. Math. Soc., 25(1):123-150, 2012. With an appendix by Brian Conrad.

[22] C. Debry. Towards a class number formula for Drinfeld modules. PhD thesis, University of Amsterdam / KU Leuven (available at http://hdl.handle.net/11245/1.545161), November 2016.

[23] F. Demeslay. A class formula for $L$-series in positive characteristic. arXiv:1412.3704, 2014.

[24] F. Demeslay. Formules de classes en caractéristique positive. PhD thesis, Université de Caen Normandie, October 2015.

[25] V. Drinfeld. Elliptic modules, Math. Sbornik 94 (1974), 594-627. Math. U.S.S.R. Sbornik, 23:561-592, 1976.

[26] V. Drinfeld. Elliptic modules II. Math. U.S.S.R. Sbornik, 31:159-170, 1977. 
[27] V. Drinfeld. Varieties of modules of F-sheaves. Functional Analysis and its Applications, 21:107-122, 1987.

[28] V. Drinfeld. Cohomology of compactified manifolds of modules of $F$-sheaves of rank 2. Journal of Soviet Mathematics, 46:1789-1821, 1989.

[29] J. Fang. Special $L$-values of abelian $t$-modules. J. Number Theory, 147:300-325, 2015.

[30] E.-U. Gekeler. On finite Drinfeld modules. J. Algebra, 141:187-203, 1991.

[31] D. Goss. Basic Structures of function field arithmetic, volume 35 of Ergebnisse der Mathematik und ihrer Grenzgebiete (3). Springer-Verlag, Berlin, 1996.

[32] N. Green. Special zeta values using tensor powers of Drinfeld modules. arXiv:1706.06048, 2017.

[33] N. Green. Tensor powers of rank 1 Drinfeld modules and periods. arXiv:1706.03854, 2017.

[34] N. Green and M. Papanikolas. Special $L$-values and shtuka functions for Drinfeld modules on elliptic curves. Res. Math. Sci. (to appear), arXiv:1607.04211, 2016.

[35] U. Hartl and S. Hüsken. A criterion for good reduction of Drinfeld modules and Anderson motives in terms of local shtukas. Ann. Sc. Norm. Super. Pisa Cl. Sci. (5), 15:25-43, 2016.

[36] U. Hartl and A. K. Juschka. Pink's theory of Hodge structures and the Hodge conjectures over function fields. In G. Böckle, D. Goss, U. Hartl, and M. Papanikolas, editors, Proceedings of the conference on "t-motives: Hodge structures, transcendence and other motivic aspects", BIRS, Banff, Canada 2009, page arXiv : 1607.01412. European Mathematical Society, 2016.

[37] D. Hayes. Explicit class field theory in global function fields. In Studies in algebra and number theory, volume 6 of Adv. in Math. Suppl. Stud., pages 173-217. Academic Press, New YorkLondon, 1979.

[38] D. Hayes. A brief introduction to Drinfeld modules. In The arithmetic of function fields (Columbus, OH, 1991), volume 2 of Ohio State Univ. Math. Res. Inst. Publ., pages 1-32. de Gruyter, Berlin, 1992.

[39] L. Lafforgue. Chtoucas de Drinfeld et conjecture de Ramanujan-Petersson. Astérisque, 243, 1997.

[40] L. Lafforgue. Chtoucas de Drinfeld et correspondance de Langlands. Invent. Math., 147:1-241, 2002.

[41] V. Lafforgue. Valeurs spéciales des fonctions $L$ en caractéristique $p$. J. Number Theory, 129:2600-2634, 2009.

[42] V. Lafforgue. Chtoucas pour les groupes réductifs et paramétrisation de Langlands globale. J. Amer. Math. Soc., 31(3):719-891, 2018.

[43] G. Laumon, M. Rapoport, and U. Stuhler. D-elliptic sheaves and the Langlands correspondence. Invent. Math., 113:217-338, 1993.

[44] B. Matthias and U. Hartl. Pure Anderson motives and abelian $\tau$-sheaves. Math. Z., 268(12):67-100, 2011.

[45] M. Mornev. Shtuka cohomology and special values of Goss L-functions. PhD thesis, University of Amsterdam (available at http://hdl.handle.net/1887/61145), February 2018.

[46] M. Papanikolas. Log-algebraicity on tensor powers of the Carlitz module and special values of Goss $L$-functions. work in progress, 167 pages (last version: 28 April 2015).

[47] M. Papanikolas. Tannakian duality for Anderson-Drinfeld motives and algebraic independence of Carlitz logarithms. Invent. Math., 171(1):123-174, 2008.

[48] F. Pellarin. Values of certain $L$-series in positive characteristic. Ann. of Math., 176(3):20552093, 2012.

[49] S. Sinha. Periods of $t$-motives and transcendence. Duke Math. J., 88(3):465-535, 1997.

[50] L. Taelman. Artin $t$-motives. J. Number Theory, 129:142-157, 2009.

[51] L. Taelman. Special $L$-values of $t$-motives: a conjecture. Int. Math. Res. Not., 2009(16):2957$2977,2009$.

[52] L. Taelman. A Dirichlet unit theorem for Drinfeld modules. Math. Ann., 348(4):899-907, 2010.

[53] L. Taelman. A Herbrand-Ribet theorem for function fields. Invent. Math., 188:253-275, 2012.

[54] L. Taelman. Special $L$-values of Drinfeld modules. Ann. of Math., 175(1):369-391, 2012.

[55] Y. Taguchi and D. Wan. L-functions of $\phi$-sheaves and Drinfeld modules. J. Amer. Math. Soc., 9(3):755-781, 1996.

[56] D. Thakur. Drinfeld modules and arithmetic in function fields. Int. Math. Res. Not., 1992(9):185-197, 1992.

[57] D. Thakur. Shtukas and Jacobi sums. Invent. Math., 111:557-570, 1993. 
[58] D. Thakur. Function field arithmetic. World Scientific Publishing Co., Inc., River Edge, NJ, 2004.

[59] D. Thakur. Multizeta in function field arithmetic . In G. Böckle, D. Goss, U. Hartl, and M. Papanikolas, editors, Proceedings of the conference on "t-motives: Hodge structures, transcendence and other motivic aspects", BIRS, Banff, Canada 2009. European Mathematical Society, 2016.

[60] L. I. Wade. Certain quantities transcendental over $G F\left(p^{n}, x\right)$. Duke Math. J., 8:701-720, 1941.

[61] J. Yu. Transcendence and special zeta values in characteristic p. Ann. of Math. (2), 134(1):123, 1991

[62] J. Yu. Analytic homomorphisms into Drinfeld modules. Ann. of Math. (2), 145(2):215-233, 1997.

Université de Caen Normandie, Laboratoire de Mathématiques Nicolas Oresme, CNRS UMR 6139, Campus II, Boulevard Maréchal Juin, B.P. 5186, 14032 Caen Cedex, France.

E-mail address: bruno.angles@unicaen.fr

CNRS - Université de Caen Normandie, laboratoire de Mathématiques Nicolas Oresme, CNRS UMR 6139, Campus II, Boulevard Maréchal Juin, B.P. 5186, 14032 Caen Cedex, FRANCE.

E-mail address: tuan.ngodac@unicaen.fr

Université de Caen Normandie, laboratoire de Mathématiques Nicolas Oresme, CNRS UMR 6139, Campus II, Boulevard Maréchal Juin, B.P. 5186, 14032 Caen Cedex, France.

E-mail address: floric.tavares-ribeiro@unicaen.fr 